\title{
Associação entre Estado Nutricional e Força de Preensão Manual em Pacientes Oncológicos em Cuidados Paliativos
}

https://doi.org/10.32635/2176-9745.RBC.2018v64n4.196

\author{
Association Between Nutritional Status and Manual Grip Strength in Oncologic Patients in Palliative Care \\ Asociación entre Estado Nutricional y Fuerza de Asimiento Manual en Pacientes Oncológicos en Cuidados Paliativos
}

Eliza Helena Eliete da Silva'; Florença Maria Borges²; Franciele Carolina Soares da Cruz ${ }^{3}$; Geórgia das Graças Pena ${ }^{4}$

Resumo

Introduçáo: $\mathrm{O}$ acompanhamento nutricional nos cuidados paliativos é de extrema importância, já que os níveis de mortalidade, desnutrição e perda de peso não intencional ocorre na maioria dos pacientes oncológicos. Dessa maneira, é possível prevenir carências nutricionais e perdas de peso, o que leva a uma piora do estado nutricional. Objetivo: $\mathrm{O}$ objetivo do estudo foi avaliar a associação entre a classificação do estado nutricional obtido pela avaliação subjetiva global produzida pelo próprio paciente (ASG-PPP), a força de preensão manual (FPM) e a espessura do músculo adutor do polegar (EMAP) em pacientes oncológicos em cuidados paliativos exclusivos. Método: Foram avaliados 70 pacientes oncológicos cadastrados no Programa de Cuidados Paliativos. Para a avaliaçáo do estado nutricional, foi utilizada a ASG-PPP. Para avaliar a associação do estado nutricional com outras medidas preditoras, foram realizadas as medidas da FPM com um dinamômetro e a EMAP com um adipômetro. Foram utilizadas estatísticas descritivas e a análise de regressão linear para avaliar a capacidade dessas medidas de predizer o estado nutricional. Resultados: Dos 70 pacientes, com idade entre 31 e 101 anos, 58,6\% eram do sexo masculino e foi encontrada frequência de desnutrição $(B+C)$ em 87,2\% ( $n=61)$ de acordo com a ASG-PPP. O valor da EMAP estava alterado em 72,9\% (n=51) pacientes e 42,9\% $(n=30)$ na FPM. A FPM foi associada ao ASG-PPP $(\beta=-0,273$; $p=0,04)$ e o EMAP não $(\beta=-0,124 ; \mathrm{p}=0,546)$; portanto, somente o FPM foi um bom preditor do estado nutricional neste estudo. Conclusáo: Sugere-se a utilização da FPM para avaliação do estado nutricional em pacientes oncológicos paliativos.

Palavras-chave: Cuidados Paliativos; Neoplasias; Estado Nutricional; Desnutrição; Força Muscular.

\begin{abstract}
Introduction: Nutritional monitoring in palliative care is extremely important, since levels of mortality, malnutrition and unintentional weight loss occur in the majority of cancer patients. In this way, it is possible to prevent nutritional deficiencies and weight loss, which leads to a worsening nutritional status. Objective: The aim of the study was to evaluate the association between the classification of nutritional status obtained by the patient-generated subjective global assessment (PG-SGA), handgrip strength (HGS) and adductor pollicis muscle (APM) in cancer patients palliative. Method: We evaluated 70 cancer patients enrolled in the exclusive palliative care program. The PG-SGA was used to assess the nutritional status. To evaluate the association of nutritional status with other predictive measures, HGS measurements were performed with a dynamometer and APM with an adipometer. Descriptive statistics and linear regression analysis were used to evaluate the ability of these measures to predict nutritional status. Results: Of the 70 patients, aged between 31 and 101 years, in which $58.6 \%$ were male and malnutrition $(\mathrm{B}+\mathrm{C})$ was found in $87.2 \%(\mathrm{n}=61)$ according to PG-SGA. The value of APM was altered in $72.9 \%(\mathrm{n}=51)$ patients and $42.9 \%(\mathrm{n}=30)$ in HGS. HGS was associated with PG-SGA $(b=-0.273$, $\mathrm{p}=0.04)$ and APM didn't $(\mathrm{b}=-0,124 ; \mathrm{p}=0.546)$; therefore, only HGS was a good predictor of nutritional status in this study. Conclusion: It's suggested the use of HGS to evaluate nutritional status in palliative cancer patients. Key words: Palliative Care; Neoplasms; Nutritional Status; Malnutrition; Muscle Strength.
\end{abstract}

\section{Resumen}

Introducción: El seguimiento nutricional en los cuidados paliativos es de extrema importancia, ya que los niveles de mortalidad, desnutrición y pérdida de peso no intencional ocurre en la mayoría de los pacientes oncológicos. De esta manera, es posible prevenir carencias nutricionales y pérdidas de peso, lo que lleva a un empeoramiento del estado nutricional. Objetivo: El objetivo del estudio fue evaluar la asociación entre la clasificación del estado nutricional obtenido por la valoración subjetiva global generada por el Paciente (GP-VSG), la fuerza de asimiento manual (FAM) y el espesor del músculo aductor del pollicis (EMAP) en pacientes oncológicos paliativos exclusivos. Método: Se evaluaron 70 pacientes oncológicos registrados en el Programa de Cuidados Paliativos. Para la evaluación del estado nutricional se utilizó la GP-VSG.Para evaluar la asociación del estado nutricional con otras medidas preditoras, se realizaron las medidas de la FAM con un dinamómetro y EMAP con un adipómetro. Se utilizaron estadísticas descriptivas y análisis de regresión lineal para evaluar la capacidad de esas medidas de predecir el estado nutricional. Resultados: De los 70 pacientes, con edad entre 31 y 101 ańos, en que $58,6 \%$ eran del sexo masculino y se encontró frecuencia de desnutrición $(\mathrm{B}+\mathrm{C})$ en el 87,2\% $(\mathrm{n}=61)$ de acuerdo con la GP-VSG. El valor del EMAP estaba alterada en el $72,9 \%(n=51)$ pacientes y el $42,9 \%$ $(n=30)$ en la FAM. La FAM fue asociada al GP-VSG $(b=-0,273, p=0,04)$ y el EMAP no $(b=-0,124, p=0,546)$, por lo tanto, sólo el FAM fue un buen predictor del estado nutricional en este estudio. Conclusión: Se sugiere la utilización de la FAM para evaluación del estado nutricional en pacientes oncológicos paliativos.

Palabras clave: Cuidados Paliativos; Neoplasias; Estado Nutricional; Desnutrición; Fuerza Muscular.

\footnotetext{
${ }^{1}$ Universidade Federal de Uberlândia (UFU). Uberlândia (MG), Brasil. Orcid iD: https://orcid.org/0000-0002-1542-4629

2 UFU. Uberlândia (MG), Brasil. Orcid iD: https://orcid.org/0000-0002-8024-136X

${ }^{3}$ UFU. Uberlândia (MG), Brasil. Orcid iD: https://orcid.org/0000-0003-0869-9154

${ }^{4}$ UFU. Uberlândia (MG), Brasil. Orcid iD: https://orcid.org/0000-0002-0360-223X

Endereço para correspondência: Geórgia das Graças Pena. Av. Pará, 1720 - Umuarama. Uberlândia (MG), Brasil. CEP 38405-320. E-mail: georgia@ufu.br
} 


\section{INTRODUÇÃO}

Quando um paciente entra em cuidados paliativos é porque o câncer está na fase em que as terapias, que promovem a cura, não possuem mais o efeito esperado, elevando os níveis de mortalidade. O propósito dessa linha de cuidados é melhorar a condição de vida desse paciente ${ }^{1}$.

O serviço de assistência nutricional nos cuidados paliativos tem como objetivo prevenir as carências nutricionais, certificar-se de que o paciente mantenha o estado nutricional evitando perdas de peso e medidas antropométricas, possibilitar a modulação de sintomas e efeitos colaterais em razáo dos tratamentos, proporcionar a sensação de prazer no paciente ao se alimentar, e diminuir os níveis de mortalidade por causa da doença ${ }^{2}$.

Para fazer esse acompanhamento nutricional em pacientes com câncer em cuidados paliativos, preconiza-se o uso da avaliação subjetiva global produzida pelo próprio paciente (ASG-PPP) ${ }^{3}$; porém, um dos desafios durante a avaliaçấo do estado nutricional do paciente é que os métodos, quando usados separados, de forma isolada, levam a uma avaliação inadequada ${ }^{4}$. Ao se utilizarem diferentes parâmetros de forma combinada para a avaliação do estado nutricional do paciente, é possível que seja otimizada a triagem de pacientes oncológicos, tornando-a mais sensível na detecção de desnutrição ${ }^{5}$.

É notável que a perda de massa magra leva à perda da capacidade funcional; assim, a avaliação por meio do dinamômetro é o mais recomendado na prática para fazer essa mensuração de forma a detectar precocemente o déficit nutricional ${ }^{6}$.

Portanto, a força de preensão manual (FPM) é útil para determinar, durante a avaliação nutricional, se a desnutrição está instaurada, uma vez que a desnutrição afeta de modo direto os músculos ${ }^{7}$, levando a uma redução na força muscular do paciente ${ }^{8}$.

Outro parâmetro para estimar a perda muscular e consequentemente avaliar o estado nutricional nesses pacientes é a espessura do músculo adutor do polegar $(\mathrm{EMAP})^{9}$. A utilização do músculo adutor do polegar é indicada por sua anatomia ser bastante precisa em razão de estar localizado entre dois ossos. Além do mais, é uma técnica não invasiva, de mensuração rápida e com baixo custo. Quando inicia no paciente o processo de desnutrição, o músculo adutor do polegar apresenta uma atrofia muito importante, podendo, assim, ser utilizado para avaliação do estado nutricional e a evolução clínica dos pacientes ${ }^{10}$.

Em vista disso, é perceptível como um paciente oncológico pode ter seu estado nutricional prejudicado e o quanto isso pode levá-lo a um mau prognóstico. Em contrapartida, verifica-se que uma triagem rápida e uma eficaz avaliação do estado nutricional nesses pacientes são imprescindíveis ${ }^{11}$.

Embora a literatura demonstre muitos dados a respeito da desnutriçáo em pacientes hospitalizados e em diversas condiçôes clínicas, ainda são raros os estudos com pacientes paliativos, incluindo a avaliação da FPM e da EMAP. Diante do exposto, o objetivo do estudo foi avaliar a associação entre a classificação do estado nutricional obtido pelas ASG-PPP, FPM e EMAP em pacientes oncológicos em cuidados paliativos exclusivos.

\section{MÉTODO}

Trata-se de um estudo transversal, no período de julho a novembro de 2016, com pacientes oncológicos cadastrados no Programa de Cuidados Paliativos (PCP) do Hospital do Câncer de Uberlândia. O projeto de pesquisa foi submetido e aprovado pelo Comitê de Ética em Pesquisa da Universidade Federal de Uberlândia, sob o número CAAE 54864316.8.0000.5152 e seguiu as normas do Conselho Nacional em Saúde, contidas nas resoluçóes números 466/12 e 251/97. Os pacientes cadastrados no PCP foram convidados a participar da pesquisa e, em seguida, foi esclarecido o seu propósito, e solicitada a assinatura do Termo de Consentimento Livre e Esclarecido.

A população foi composta por 92 pacientes que perfazem 100\% dos pacientes cadastrados no PCP, cujos critérios de inclusão foram: idade superior a 18 anos, ambos os sexos e estar cadastrado no PCP. Os critérios de exclusão foram: rebaixamento do nível de consciência, confusão mental, Doença de Alzheimer relatada no prontuário e idade inferior a 18 anos. Da população total, foram excluídos, de acordo com os critérios citados, 22 pacientes $(23,9 \%)$, totalizando 70 pacientes ao final do estudo.

Foram coletadas variáveis socioeconômicas e clínicas (tempo de cadastro no PCP, ano de diagnóstico, localização do tumor primário e tratamentos já realizados por meio de um questionário previamente estruturado). Quando o paciente não sabia relatar as informaçôes, citadas anteriormente, referentes à doença, elas eram obtidas pelo prontuário médico.

Para a avaliação do estado nutricional, utilizou-se a ASG-PPP na versão traduzida para o português e validada por Gonzalez et al. ${ }^{12}$. Após preencher o formulário, o estado nutricional do paciente foi classificado em três categorias: A (bem nutrido); B (moderadamente desnutrido ou suspeito de desnutrição); ou C (gravemente desnutrido $)^{12}$.

Esse instrumento leva em consideração a alteração involuntária de peso, sintomas do trato gastrointestinal, exame físico, estresse metabólico e aspectos do tratamento, sendo recomendado durante a assistência nutricional em 
oncologia para detecção precoce do risco nutricional ou da desnutriçãoo ${ }^{12}$.

Para verificar a associação do estado nutricional com outras medidas preditoras, foi realizada avaliação antropométrica, por meio do peso, dobra cutânea tricipital (DCT), EMAP e FPM. Posteriormente, foi calculado o índice de massa corporal (IMC) $/ \mathrm{kg} / \mathrm{m}^{2}$. Para coleta do peso, foi utilizada a balança eletrônica Tanita - Tanita Body Fat Monitor Scale (Modelo TBF 531 ${ }^{\circ}$, Tanita Corporation of America, Illinois). Foi considerada a altura (m) referida pelo paciente ou seu cuidador. Para a classificação do IMC, foram utilizados os pontos de corte estabelecidos para a classificação do estado nutricional, propostos para $\operatorname{adultos}^{13}$ ( $\geq 18$ e $\leq 60$ anos) e para idosos ${ }^{14}$ ( $\geq 60$ anos). A classificação da DCT foi realizada segundo critérios de Frisancho ${ }^{15}$.

Para coletar a medida do EMAP, foi utilizado o adipômetro da marca Sanny ${ }^{\circ}$, sendo a coleta dessa medida repetida por três vezes, e a média final entre elas foi considerada. Os valores da EMAP foram classificados de acordo com Bragagnolo et al. ${ }^{16}$, que consideraram a espessura $\leq 13,4 \mathrm{~mm}$ "alterada" e $>13,4 \mathrm{~mm}$ "não alterada".

Para a FPM, foi utilizado o dinamômetro da marca $\mathrm{Jamar}^{\circledR}$ hidráulico. $\mathrm{O}$ paciente permaneceu sentado confortavelmente, com os pés apoiados no chão e quadril e joelho posicionados a, aproximadamente, $90^{\circ}$ de flexáo. O ombro da mão dominante ficou aduzido e em rotação neutra, cotovelo em flexão de $90^{\circ}$, antebraço na posição neutra e punho entre 0 e $30^{\circ}$ de extensão e entre 0 a $15^{\circ}$ de adução. A mão não dominante ficou repousada sobre a coxa do mesmo lado ${ }^{17}$. Os valores da FPM dos pacientes foram demasiadamente inferiores do que os valores de referência do estudo de Bohannon et al. ${ }^{18}$. Como se trata de um estudo de base populacional, foi utilizada a mediana para classificação, considerando, para homens, a força $\leq 14$ kgf "alterada" e > 14 kgf "não alterada"; e, para mulheres, $\leq 2$ kgf "alterada" e >2 kgf "não alterada".

Os dados foram processados com dupla digitação e checagem de consistência por meio do software EpiInfo 3.5. $4^{\circ}$. As análises foram realizadas pelo pacote estatístico Software Package Statistical System 12.0 for Windows versão 17.0 (SPSS). Inicialmente, foi testada a distribuição das variáveis em normal e não normal, analisadas pelo teste Kolmogorov-Smirnov. A análise descritiva foi realizada por intermédio de média \pm desvio-padrão ou mediana (mínimo-máximo) para variáveis quantitativas e pela proporção para variáveis qualitativas; e a análise de significância estatística foi realizada pelo teste de análise de variância Anova para variáveis quantitativas e pelo teste qui-quadrado.

Foi realizada análise de regressão linear para avaliar a capacidade preditora da EMAP e da FPM quanto ao estado nutricional (escore do AGS-PPP) como variável desfecho (dependente). Foram considerados, como fatores de confusão, variáveis sociodemográficas (idade, sexo), renda familiar e IMC. Para todas as análises, foi considerada significância estatística quando $\mathrm{p} \leq 0,05$ e intervalo de confiança (IC) de 95\%.

\section{RESULTADOS}

O estudo contou com a participaçáo de 70 pacientes oncológicos em cuidados paliativos exclusivos, maiores de 18 anos; destes, 58,6\% $(\mathrm{n}=41)$ eram do sexo masculino e $41,4 \% \quad(n=29)$ do sexo feminino, $30 \%(n=21)$ foram classificados como adultos e $70 \%(\mathrm{n}=49)$ como idosos ( $\geq 60$ anos - dados não mostrados). Em relação ao tempo de cadastramento no PCP, em 68,2\% ( $\mathrm{n}=45$ ), foi inferior a seis meses. As características socioeconômicas e clínicas são apresentadas na Tabela 1.

Foram identificadas 20 diferentes localizaçóes de tumores primários, sendo que os mais presentes acometeram regióes da cabeça e pescoço, totalizando $24,3 \%(n=17)$, seguido pelos ginecológicos $21,4 \%$ $(n=15)$. A maioria dos pacientes, 49,3\% $(n=34)$, havia recebido o diagnóstico da doença há mais de dois anos. Entre os tratamentos realizados, foi encontrada maior frequência na radioterapia com $72,9 \%(n=51)$, seguida pela quimioterapia com $65,7 \%(\mathrm{n}=46)$ e, por último, o tratamento cirúrgico com 55,7\% (n=39) (Tabela 1).

De acordo com a ASG-PPP, 12,9\% $(n=9)$ foram classificados como bem nutridos, 62,9\% $(\mathrm{n}=44)$ moderadamente desnutridos e $24,3 \%(\mathrm{n}=17)$ estavam gravemente desnutridos. Considerando o IMC, 14,7\% $(\mathrm{n}=10)$ estavam com excesso de peso, 25\% $(\mathrm{n}=17)$ eutróficos e 60,3\% ( $n=41)$ estavam desnutridos. Quando classificados segundo a EMAP, 72,9\% ( $\mathrm{n}=51)$ estavam desnutridos e 27,1\% ( $\mathrm{n}=19$ ) nutridos (Tabela 2).

Conforme a classificação da FPM, 57,1\% ( $\mathrm{n}=40)$ estavam nutridos e 42,9\% ( $n=30)$ eram desnutridos; e, por fim, de acordo com a DCT, $5,7 \%(\mathrm{n}=4)$ tinham excesso de gordura, 57,1\% ( $\mathrm{n}=40)$ eram eutróficos, $11,4 \%(n=8)$ estavam em risco de desnutrição e $25,7 \%$ $(n=18)$ desnutridos. Foi encontrada maior prevalência de desnutrição nos pacientes do sexo masculino, em quase todos os indicadores do estado nutricional (Tabela 2).

Pela classificação do estado nutricional segundo a EMAP, observa-se que 89,5\% ( $\mathrm{n}=17)$ dos desnutridos encontravam-se na faixa etária de 66 a 75 anos. Já de acordo com a FPM, a faixa etária da maioria dos desnutridos era maior que 76 anos, representando $60,0 \%$ $(\mathrm{n}=12)$ da amostra. Ainda de acordo com a classificação do estado nutricional segundo a FPM, os pacientes que 
Tabela 1. Características socioeconômicas e clínicas de pacientes oncológicos em cuidados paliativos

\begin{tabular}{|c|c|c|c|c|}
\hline \multirow[b]{2}{*}{ Variáveis } & \multirow{2}{*}{$\begin{array}{c}\text { Total } \\
\%(n=70)\end{array}$} & \multicolumn{2}{|c|}{ Sexo } & \multirow{2}{*}{$\begin{array}{c}\text { Valor de } \\
\qquad \text { p }^{*}\end{array}$} \\
\hline & & $\begin{array}{c}\text { Feminino } \\
(41,4 \%, n=29)\end{array}$ & $\begin{array}{c}\text { Masculino } \\
(58,6 \%, n=41)\end{array}$ & \\
\hline \multicolumn{4}{|l|}{ Faixa etária (anos) } & 0,437 \\
\hline$<55$ & $22,9(16)$ & $56,2(9)$ & $43,8(7)$ & \\
\hline $56-65$ & $21,4(15)$ & $46,7(7)$ & $53,3(8)$ & \\
\hline $66-75$ & $27,1(19)$ & $31,6(6)$ & $68,4(13)$ & \\
\hline$>76$ & $28,6(20)$ & $35,0(7)$ & $65,0(13)$ & \\
\hline \multicolumn{4}{|l|}{ Renda familiar (salários mínimos**) } & 0,967 \\
\hline$<1$ & $3,1(2)$ & $50,0(1)$ & $50,0(1)$ & \\
\hline $1-3$ & $55,4(36)$ & $41,7(15)$ & $58,3(21)$ & \\
\hline$>3$ & $41,5(27)$ & $40,7(11)$ & $59,3(16)$ & \\
\hline \multicolumn{4}{|c|}{ Tempo de cadastro Programa de Cuidados Paliativos (meses) } & 0,440 \\
\hline$<6$ & $68,2(45)$ & $42,4(17)$ & $57,6(28)$ & \\
\hline $6-12$ & $10,6(7)$ & $42,9(3)$ & $57,1(4)$ & \\
\hline$>12$ & $21,2(14)$ & $57,1(8)$ & $42,9(6)$ & \\
\hline \multicolumn{4}{|l|}{ Tempo de diagnóstico } & 0,959 \\
\hline $1-6$ meses & $18,8(13)$ & $46,2(6)$ & $53,8(7)$ & \\
\hline 7-11 meses & $8,7(6)$ & $33,3(2)$ & $66,7(4)$ & \\
\hline 1-2 anos & $23,2(16)$ & $43,8(7)$ & $56,2(9)$ & \\
\hline$>2$ anos & $49,3(34)$ & $41,2(14)$ & $58,8(20)$ & \\
\hline \multicolumn{4}{|l|}{ Localização do tumor } & 0,005 \\
\hline Cabeça e pescoço & $24,3(17)$ & $17,3(5)$ & $22,0(9)$ & \\
\hline Ginecológicos & $21,4(15)$ & $51,7(15)$ & $0(0)$ & \\
\hline Próstata & $8,6(6)$ & $0(0)$ & $14,7(6)$ & \\
\hline Pulmão & $12,8(9)$ & $13,7(4)$ & $12,1(5)$ & \\
\hline Outros*** & $32,9(23)$ & $17,3(5)$ & $51,2(21)$ & \\
\hline \multicolumn{5}{|l|}{ Tratamento submetido } \\
\hline \multicolumn{4}{|l|}{ Radioterapia } & 0,117 \\
\hline Sim & $72,9(51)$ & $47,1(24)$ & $52,9(27)$ & \\
\hline Não & $27,1(19)$ & $26,3(5)$ & $73,7(14)$ & \\
\hline \multicolumn{4}{|l|}{ Quimioterapia } & 0,132 \\
\hline Sim & $65,7(46)$ & $47,8(22)$ & $52,2(24)$ & \\
\hline Não & $34,3(24)$ & $29,2(7)$ & $70,8(17)$ & \\
\hline \multicolumn{4}{|l|}{ Cirurgia } & 0,132 \\
\hline Sim & $55,7(39)$ & $46,2(18)$ & $53,8(21)$ & \\
\hline Não & $44,3(31)$ & 35,5 (11) & $64,5(20)$ & \\
\hline
\end{tabular}

Legendas: *Teste qui-quadrado; ${ }^{* *}$ Salário mínimo vigente no ano de 2016 R\$ 880,00; ${ }^{* * *}$ Outras localizaçóes do tumor (fígado, esôfago, colón, bexiga, glândulas adrenais e outros)

realizaram tratamento quimioterápico tiveram diferença significativa em relação aos demais tratamentos; porém, em razão da grande variação entre os tipos de cânceres, o que leva a uma grande variabilidade nas doses da quimioterapia e que pode ter sido um fator de confusão para as análises (Tabela 3).

O EMAP não foi associado à ASG-PPP, $\left(r^{2}=0,080\right.$; $b=-0,124 ; p=0,546 ; \mathrm{IC}=95 \%[-0,531$ - 0,284]), que mostrou predição em $8 \%$ em relação ao escore da ASGPPP. Porém, a FPM foi associada ao escore da ASG-PPP $\left(r^{2}=0,141 ; b=-0,273 ; p=0,04 ; \mathrm{IC}=95 \%[-0,459--0,063]\right)$, enquanto a FPM explicou $14,1 \%$ das variâncias do escore da ASG-PPP, mesmo após ajuste por idade, sexo, renda familiar e IMC (Tabela 4).

\section{DISCUSSÃO}

No presente estudo, foi possível observar que a frequência da desnutriçâao foi considerável nos diversos indicadores avaliados, sendo encontrada frequência de desnutrição de 87,2\% (n=61) de acordo com a ASG-PPP; 72,9\% ( $\mathrm{n}=51)$ segundo o EMAP; e 42,9\% $(\mathrm{n}=30)$ de acordo com a FPM. A ASG-PPP é um instrumento com alta especificidade e sensibilidade para pacientes oncológicos 
Tabela 2. Características antropométricas e do estado nutricional de pacientes oncológicos em cuidados paliativos

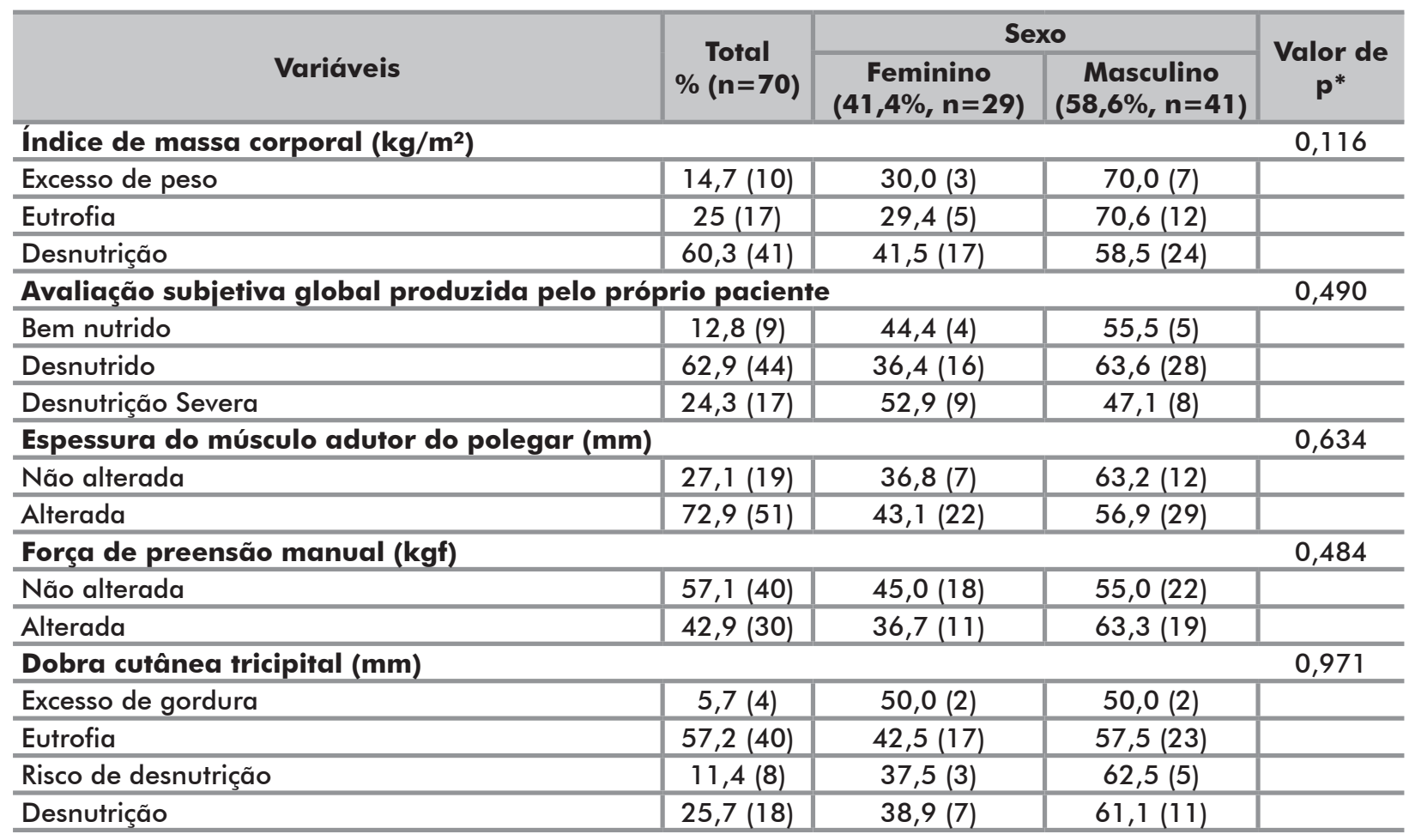

Legenda: *Teste qui-quadrado;.

por detectar sinais e sintomas específicos da doença e leva em consideração a alteração involuntária de peso, sintomas do trato gastrointestinal, exame físico e aspectos do tratamento conforme verificado por Santos et al. ${ }^{19}$.

No presente estudo, a FPM mostrou ser um instrumento que possui capacidade preditora do estado nutricional nessa população, ao passo que o EMAP mostrou o contrário. O uso da FPM explicou 14,1\% das variâncias do escore final obtido na ASG-PPP, enquanto o EMAP explicou $8 \%$.

A desnutrição nesses pacientes é decorrente da combinação de vários fatores que influenciam o estado nutricional, como a terapia escolhida e o avanço no crescimento das células tumorais. Dessa maneira, percebese a importância de combinar, ao tratamento oncológico, uma intervenção nutricional, objetivando, assim, melhora do estado nutricional e da qualidade de vida dos pacientes ${ }^{20}$.

A FPM, além de contribuir para avaliação do estado nutricional, é interessante para avaliar a capacidade funcional, sendo um bom parâmetro para verificar o prognóstico do paciente. Assim, pelo fato de o tratamento ser agressivo e invasivo, quanto mais autonomia o paciente tiver e conseguir manter suas atividades diárias, maiores serão as chances de manter o estado nutricional preservado nesses pacientes ${ }^{21}$.

Em um estudo realizado por Kilgour et al. ${ }^{22}$, com 203 pacientes oncológicos em estágio avançado, os autores verificaram que mais de $70 \%$ dos pacientes apresentaram FPM abaixo do percentil 50 e cerca de $27 \%$, abaixo do percentil 10. O grupo dos pacientes com FPM menor que o percentil 10 apresentou significativamente menor massa magra e massa gorda, em comparaçáo aos demais grupos. Ainda observaram que o resultado da FPM pode auxiliar na implementaçáo de medidas para recuperação do estado nutricional do paciente, já que a FPM foi preditora independente para a sobrevida.

Conforme o estudo realizado por Queiroz et al. ${ }^{23}$ com 210 pacientes com câncer avançado em cuidados paliativos, foi possível verificar que mais de $20 \%$ dos pacientes estavam dinapênicos (baixa força muscular - FPM < percentil 10), mais de $15 \%$ apresentaram a atrofia muscular (baixa massa muscular - adequação da circunferência muscular do braço $\mathrm{CMB}<90 \%$ ) e $32,4 \%$ dos pacientes estavam sarcopênicos. Ainda foi possível analisar que as variáveis antropométricas foram significativamente menores nos indivíduos com sarcopenia, além de a presença da sarcopenia ter sido associada a um maior escore obtido na ASG-PPP.

Outro estudo com 60 pacientes oncológicos em tratamento cirúrgico em razáo do câncer de pulmão verificou associação significativa $(p=0,01)$ entre a diminuição da FPM e a desnutrição nos pacientes, com resultados inferiores ao grupo de pacientes eutróficos ${ }^{24}$. 
Tabela 3. Características antropométricas e nutricionais de pacientes oncológicos em cuidados paliativos de acordo com as classificações de EMAP e FPM

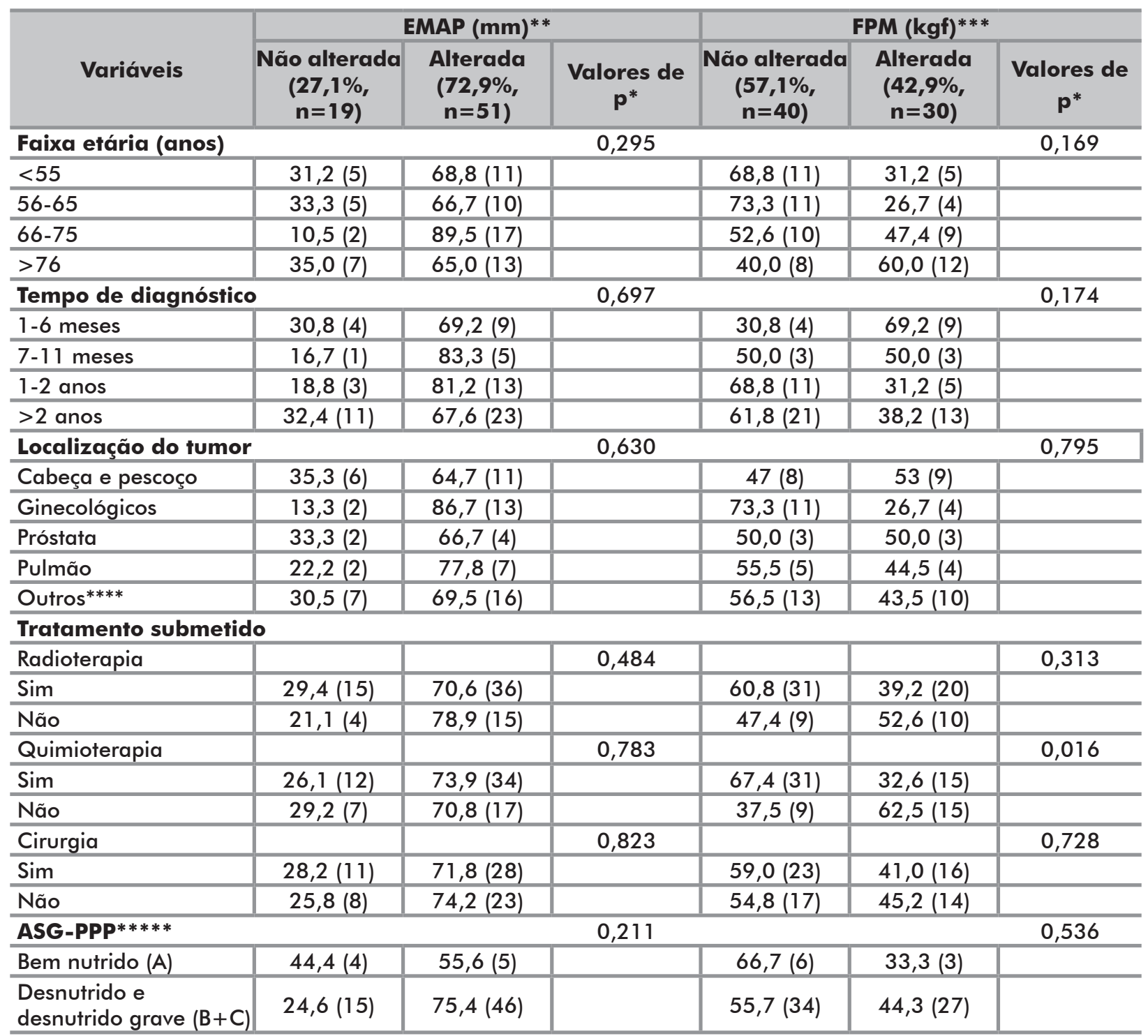

Legenda: *Teste qui-quadrado; ${ }^{* *}$ Espessura do músculo adutor do polegar; ${ }^{* * *}$ Força de preensão manual; *** Outras localizaçôes do tumor (fígado, esôfago, colón, bexiga, glândulas adrenais e outros); *****Avaliação subjetiva global produzida pelo próprio paciente.

Nota: $\mathrm{O} n$ varia de acordo com a disponibilidade do dado.

Tabela 4. Regressão linear da FPM e EMAP na associação com o escore de ASG-PPP***

\begin{tabular}{|c|c|c|c|c|c|}
\hline \multirow{2}{*}{ Variáveis } & \multirow{2}{*}{$\begin{array}{c}\mathbf{R}^{\mathbf{2}} \\
\text { Ajustado** }\end{array}$} & \multirow{2}{*}{ Beta } & \multicolumn{2}{|c|}{ Intervalo de confiança } & \multirow{2}{*}{$\begin{array}{c}\text { Valor de } \\
\text { p* }^{*}\end{array}$} \\
\hline & & & Inferior & Superior & \\
\hline \multicolumn{6}{|c|}{ Espessura do músculo adutor do polegar (mm) } \\
\hline Modelo não ajustado & 0,030 & $-0,234$ & $-0,558$ & 0,090 & 0,154 \\
\hline Modelo ajustado** & 0,080 & $-0,124$ & $-0,531$ & 0,284 & 0,546 \\
\hline \multicolumn{6}{|c|}{ Força de preensão manual (kgf) } \\
\hline Modelo não ajustado & 0,092 & $-0,261$ & $-0,459$ & $-0,063$ & 0,011 \\
\hline Modelo ajustado** & 0,141 & $-0,273$ & $-0,533$ & $-0,013$ & 0,04 \\
\hline
\end{tabular}

Legendas: *Nível de significância p<0,05; **Ajustado por idade, sexo, renda familiar, IMC; ***Variável dependente. 
Um estudo com 203 pacientes com câncer avançado demonstrou que a menor FPM estava associada com as maiores perdas de massa magra e gorda, juntamente com a maior incidência de sarcopenia e menor força isocinética do quadríceps, segundo Wallengren et al. ${ }^{25}$. Os resultados encontrados no estudo citado foram resultantes de associaçóes realizadas da perda de peso não intencional.

Nesta avaliação, de acordo com a EMAP, verificou-se que, em $72,9 \%(\mathrm{n}=51)$ dos pacientes, a desnutrição foi detectada. Por haver poucos estudos realizados com a mesma população, não foi possível comparar a incidência de desnutrição com a EMAP em pacientes oncológicos paliativos. Mas, em contrapartida, a EMAP é bastante estudada como um parâmetro para diagnóstico do estado nutricional de pacientes cirúrgicos, conforme estudo realizado por Valente et al. ${ }^{26}$, no qual, em uma amostra de 150 pacientes candidatos à cirurgia, $40 \%(\mathrm{n}=60)$ foram diagnosticados pela EMAP como desnutridos.

Como relatado anteriormente, são poucos os estudos que utilizaram tanto a EMAP quanto a FPM, e a ASG-PPP para avaliarem o estado nutricional em pacientes oncológicos paliativos. O que impossibilitou uma maior comparação dos resultados com os achados com a literatura, verificando se iriam ser encontrados resultados semelhantes em outros estudos realizados com pacientes oncológicos paliativos.

De acordo com a regressão linear, ao associar o valor da EMAP com o escore final da ASG-PPP, não se encontrou associação significativa nos modelos não ajustado e ajustado por sexo, idade, renda familiar e IMC. Não foi verificado nenhum estudo que tenha realizado a mesma análise em pacientes oncológicos em cuidados paliativos.

Em contrapartida, em um estudo com 110 pacientes cirúrgicos, no qual a indicaçáo de cirurgia em 33,6\% $(\mathrm{n}=37)$ era em virtude das neoplasias, realizado por Valente et al. ${ }^{26}$, foi concluído que a EMAP é um método eficiente na detecção de desnutrição. Portanto, o EMAP parece ser um bom preditor em alguns grupos de pacientes; mas, na área de cuidados paliativos oncológicos, seria necessária a realização de mais estudos e com uma amostragem maior.

Entretanto, quando se relaciona a FPM com o escore final da ASG-PPP, encontra-se associação significativa e negativa, mesmo quando ajustada por sexo, idade, renda familiar e IMC. Os resultados estão de acordo com estudo de Flood et al. ${ }^{27}$, que constataram que a FPM pode prever independentemente o estado nutricional e a mudança no estado nutricional definido pelo escore e ASG-PPP em um grupo heterogêneo de pacientes internados em um hospital, concluindo, assim, que o uso da FPM pode fornecer rápidas informaçóes relacionadas com o estado nutricional de forma não subjetiva e não invasiva.

Como relatado por Bauer et al..$^{28}$, utilizar a FPM para monitorar o estado nutricional de pacientes oncológicos pode ser mais vantajoso do que a ASG-PPP, em razão da necessidade de saber o peso atual do paciente. Dessa maneira, o nutricionista pode realizar a avaliaçáo do estado nutricional do paciente, mesmo sem o valor de peso. Assim, realizando uma avaliação precisa e iniciando a terapia nutricional quando necessária.

Foi observado no estudo que, de acordo com a ASG-PPP, a maioria dos pacientes em cuidados paliativos foi classificada como desnutridos nos indicadores utilizados, o que é esperado na assistência nutricional em pacientes que possuem câncer em um estágio avançado. O estudo realizado por Marin Caro et al. ${ }^{29}$ obteve resultado semelhante no qual cerca de $81 \%$ dos pacientes apresentaram desnutrição. Já em outro estudo realizado por Kwang, Kandiah ${ }^{30}$, aproximadamente 70\% da população apresentaram desnutrição, ambos os estudos foram realizados em pacientes oncológicos paliativos.

Segundo o Consenso Nacional de Nutrição Oncológica ${ }^{3}$, durante a avaliaçáo do estado nutricional de pacientes em cuidados paliativos, devem ser evitados quaisquer métodos que levem ao desconforto tanto físico quanto emocional. Como a dinamometria é um método não invasivo e é citada para avaliação no paciente oncológico - combinada com a NRS-2002 (avaliação de risco nutricional), ASG ou ASG-PPP em paciente adulto em tratamento clínico (quimioterapia e radioterapia) -, o presente estudo sugere a ampliação de seu uso para predizer a desnutrição também em pacientes em cuidados paliativos.

Algumas limitaçóes devem ser consideradas, como a causalidade reversa do desenho transversal, não sendo possível estabelecer a relação causa-efeito dos resultados observados, bem como grande parte da amostra ter menos de seis meses no PCP. Além disso, apesar de se obter a populaçáo total dos pacientes cadastrados nos cuidados paliativos, deve-se ter cautela na extrapolação dos dados para outras populaçóes no mesmo contexto, considerando também a falta de padrão de referência de parâmetros antropométricos e de FPM específicos para indivíduos com câncer avançado em cuidados paliativos.

\section{CONCLUSÃO}

Sugere-se a utilização da FPM como método prático, de baixo custo e não invasivo para predição do estado nutricional em pacientes oncológicos paliativos, sendo uma grande vantagem, particularmente, para os países em desenvolvimento.

\section{CONTRIBUIÇÕES}

Eliza Helena Eliete da Silva contribuiu na coleta, elaboração do banco de dados e revisão crítica e aprovação 
da versão final. Florença Maria Borges contribuiu na coleta e elaboraçáo do banco de dados. Franciele Carolina Soares da Cruz contribuiu na concepção do estudo, coleta e análise dos dados. Geórgia das Graças Pena contribuiu na concepção do estudo, na análise dos dados e revisão crítica e aprovação da versão final.

\section{DECLARAÇÃO DE CONFLITO DE INTERESSES}

Nada a declarar.

\section{FONTES DE FINANCIAMENTO}

Não há.

\section{REFERÊNCIAS}

1. World Health Organization. National cancer control programmes: policies and managerial guidelines. 2nd ed. Geneva: WHO; 2002.

2. Acreman $S$. Nutrition in palliative care. Br J Community Nurs. 2009;14(10):427-431. doi: https://doi. org/10.12968/bjen.2009.14.10.44494.

3. Instituto Nacional de Câncer José Alencar Gomes da Silva. Consenso nacional de nutriçáo oncológica. 2 ed. Rio de Janeiro: INCA; 2015.

4. Ryu SW, Kim IH. Comparison of different nutritional assessments in detecting malnutrition among gastric cancer patients. World J Gastroenterol. 2010;16(26):3310-7. doi: https://doi.org/10.3748/wjg.v16.i26.3310.

5. Faramarzi E, Mahdavi R, Mohammad-Zadeh M, Nasirimotlagh B. Validation of nutritional risk index method against patient-generated subjective global assessment in screening malnutrition in colorectal cancer patients. Chin J Cancer Res. 2013;25(5):544-548. doi: https://doi.org/10.3978/j.issn.1000-9604.2013.10.04.

6. Fearon K, Strasser F, Anker SD, Bosaeus I, Bruera E, Fainsinger RL, et al. Definition and classification of cancer cachexia: an international consensus. Lancet Oncol. 2011;12(5):489-95. doi: https://doi.org/10.1016/ S1470-2045(10)70218-7.

7. Humphreys J, Maza P, Hirsch S, Barrera G, Gattas V, Bunout D. Muscle strength as a predictor of loss of functional status in hospitalized patients. Nutrition 2002;18(7-8):616-20. doi: https://doi.org/10.1016/ S0899-9007(02)00756-6.

8. Norman K, Stobäus N, Gonzalez MC, Schulzke JD, Pirlich M. Hand grip strength: outcome predictor and marker of nutritional status. Clin Nutr. 2011;30(2):13542. doi: https://doi.org/10.1016/j.clnu.2010.09.010.

9. Freitas BJSA, Mesquita LC, Teive NJV, Souza SR. Antropometria clássica e músculo adutor do polegar na determinação do prognóstico nutricional em pacientes oncológicos. Rev Bras Cancerol. 2010;56(4):415-22.
10. Poziomyck AK, Corleta OC, Cavazzola LT, Weston AC, Lameu EB, Coelho LJ, et al. Adductor pollicis muscle thickness and prediction of postoperative mortality in patients with stomach cancer. ABCD, Arq Bras Cir Dig. 2018;31(1):e1340. doi: https://doi.org/10.1590/0102$672020180001 \mathrm{e} 1340$.

11. Oliveira T. A importância do acompanhamento nutricional para pacientes com câncer. Prát Hosp. 2007;9(51):150-4.

12. Gonzalez MC, Duarte RRP, Budziareck MB. Adductor pollicis muscle: reference values of its thickness in a healthy population. Clin Nutr. 2010;29(2):268-71. doi: https://doi.org/10.1016/j.clnu.2009.08.012

13. World Health Organization. Physical status: the use and interpretation of anthropometry: report of a WHO Expert Committee. Geneva: World Health Organization; 1995. (WHO Technical Report Series, 854).

14. Lipschitz DA. Screening for nutritional status in the elderly. Prim Care. 1994 Mar;21(1):55-67.

15. Frisancho AR. New norms of upper limb fat and muscle areas for assessment of nutritional status. Am J Clin Nutr. 1981;34(11):2540-5. doi: https://doi.org/10.1093/ ajcn/34.11.2540.

16. Bragagnolo R, Caporossi FSI, Dock-Nascimento DB, Aguilar-Nascimento JE. Espessura do músculo adutor do polegar: um método rápido e confiável na avaliação nutricional de pacientes cirúrgicos. Rev Col Bras Cir. 2009;36(5):371-376. doi: http://dx.doi.org/10.1590/ S0100-69912009000500003.

17. Fess EE. Grip strength. In: American Society of Hand Therapists. Clinical assessment recommendations. 2nd ed. Chicago: American Society of Hand Therapists; 1992. p.41-5.

18. Bohannon RW, Peolsson A, Massy-Westropp N, Desrosiers J, Bear-Lehman J. Reference values for adult grip strength measured with a Jamar dynamometer: a descriptive meta-analysis. Physiotherapy, 2006;92(1):1115. doi: https://doi.org/10.1016/j.physio.2005.05.003.

19. Santos DM, Alvarenga MC, Sala PC, Dias MCG. Avaliação subjetiva global do estado nutricional produzida pelo paciente (ASG-PPP). Nutr Pauta 2009;17(96):29-33.

20. Instituto Nacional de Câncer. Consenso nacional de nutrição oncológica. Rio de Janeiro: INCA; 2009.

21. Yavuzsen T, Walsh D, Davis MP, Kirkova J, Jin T, LeGrand S, et al. Components of the anorexia-cachexia syndrome: gastrointestinal symptoms correlates of cancer anorexia. Support Care Cancer. 2009;17(12):1531-41. doi: https://doi.org/10.1007/s00520-009-0623-5.

22. Kilgour RD, Vigano A, Trutschnigg B, Lucar E, Borod M, Morais JA. Handgrip strength predicts survival and is associated with markers of clinical and functional outcomes in advanced cancer patients. Support Care 
Cancer. 2013;21(12):3261-70. doi: https://doi. org/10.1007/s00520-013-1894-4.

23. Queiroz MSC, Wiegert EVM, Lima LC, Oliveira LC. Associação entre sarcopenia, estado nutricional e qualidade de vida em pacientes com câncer avançado em cuidados paliativos. Rev Bras Cancerol. 2018;64(1):6975.

24. Piskorz L, Lesiak T, Brocki M, Klimek-Piskorz E, Smigielski J, Misiak P, et al. Biochemical and functional indices of malnutrition in patients with operable, nonmicrocelullar lung cancer. Nutr Hosp. 2011;26(5):102532. doi: 10.3305/nh.2011.26.5.5170.

25. Wallengren O, Lundholm K, Bosaeus I. Diagnostic criteria of cancer cachexia: relation to quality of life, exercise capacity and survival in unselected palliative care patients. Support Care Cancer. 2013;21(6):1569-77. https://doi.org/10.1007/s00520-012-1697-z.

26. Valente KP, Silva NMF, Faioli AB, Barreto MA, de Moraes RAG, Guandalini VR. Thickness of the adductor pollicis muscle in nutritional assessment of surgical patients. Einstein (São Paulo). 2016;14(1):18-24. doi: http://dx.doi.org/10.1590/S1679-45082016AO3596.

27. Flood A, Chung A, Parker H, Kearns V, O’Sullivan TA. The use of hand grip strength as a predictor of nutrition status in hospital patients. Clin Nutr. 2014;33(1):10614. doi: https://doi.org/10.1016/j.clnu.2013.03.003.

28. Bauer J, Capra S, Ferguson M. Use of the scored PatientGenerated Subjective Global Assessment (PG-SGA) as a nutrition assessment tool in patients with cancer. Eur J Clin Nutr 2002;56(8):779-85.

29. Marín Caro MM, Gómez Candela C, Castillo Rabaneda R, Lourenço Nogueira T, García Huerta M, Loria Kohen V. et al. Nutritional risk evalution and establishment of nutritional support in oncology patients according to the protocol of the Spanish Nutrition and Cancer Group. Nutr. Hosp. 2008;23(5):458-68.

30. Kwang AY, Kandiah M. Objective and subjective nutritional assessment of patients with cancer in palliative care. Am J Hosp Palliat Care. 2010;27(2):117-26. doi: https://doi.org/10.1177/1049909109353900. 